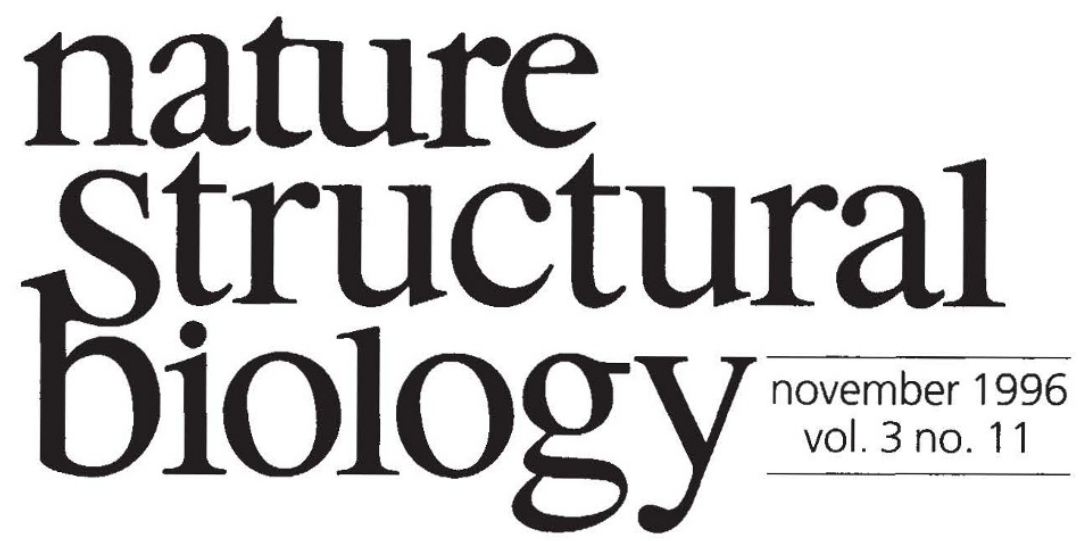

\title{
Hold your enemies closer
}

On October 7, the Nobel prize in medicine was awarded to Peter C. Doherty and Rolf M. Zinkernagel for their insight into the nature of cellular immunity. Their conclusions about the key molecules involved were not based on direct experiments on the proteins themselves. Rather, their most important conclusions rested on an experiment in which T-cells from an immunized mouse killed cells infected with a virus, but only if those cells came from the same mouse (or the same strain). They reasoned that the T-cells needed two molecular pieces of information to identify an infected cell to be killed: not only did an appropriate antigen have to be present, but also the major histocompatibility complex (MHC) that identified the infected cells as belonging to the same individual as the T-cells ${ }^{1}$.

The molecules implicated by Zinkernagel and Doherty's ideas are after twenty years yielding to structural research. These are the T-cell receptor (TCR) complex, and its target, the MHC in complex with antigen. The MHC presents peptide antigens derived from foreign invaders, usually a virus. T-cells perceive this ensemble through the TCR. Helper T-cells recognize class II MHC on the surface of antigen presenting cells, resulting in T-cell proliferation and also activation and consequent stimulation of other cells of the immune system involved in both the cellular and humoral response. Cytotoxic T-cells (CTL) recognize antigen presented by class I MHC, first as a signal for final differentiation, and later in recognition and lysis of an infected cell that posts the same antigen on its own MHC, a flag for that cell's destruction.

The MHC is giving up its structural secrets ${ }^{2}$, but direct structural information was not available on how TCR recognizes MHC with antigen presented. This has been remedied, thanks to the recent X-ray crystal structure of a TCR, along with the structure of its complex with MHC and antigen, described by K.C. Garcia et $a .^{3}$. The TCR is composed of two chains (there are two forms of TCR, an $\alpha \beta$ chain form-by far the most common-and a $\gamma \delta$ form: Garcia et al. have solved the former). As a member of the immunoglobulin superfamily, TCR shares many recognizable features: the TCR has constant $(\mathrm{C})$ and variable $(\mathrm{V})$ domains; further, the genes encoding the TCR chains undergo VDJ recombination to generate diversity in a way very similar to that which generates antibody diversitythough the TCR repertoire is not as great. From sequence similarity with antibodies (30-70\%), as well as previous structural studies of individual $\alpha$ - and $\beta$-chains, it was expected that TCR and immunoglobins would have common structural features, perhaps extending to the way they recognized targets. 


\section{IMAGE \\ UNAVAILABLE \\ FOR \\ COPYRIGHT \\ REASONS}

Fig. 1 The extracellular domain of TCR. The $\alpha$-chain is light brown and the $\beta$-chain is green. The four domains are labelled, and the CDR loops are at the top of the figure. The native TCR is glycosylated; the N-linked carbohydrates in the structure are in grey. One of the $C \alpha$ carbohydrates is in a position to interact with the $C \beta$ domain.

\section{IMAGE UNAVAILABLE FOR COPYRIGHT REASONS}

Fig. 2 A view of the complex. TCR is coloured as in Fig. 1 (but oriented with the $V$ domains downward), the bound peptide antigen is in red, and $\mathrm{MHC}$ is in blue-green. The CDR3s penetrate the groove formed by two helices on the MHC. Figures kindly supplied by K.C. Garcia.
The $2.5 \AA$ structure of the TCR extracellular domain (Fig. 1$)^{3}$ reveals two chains, each composed of two domains $(\mathrm{V} \alpha, \mathrm{C} \alpha ; \mathrm{V} \beta, \mathrm{C} \beta)$, and shows overall similarity with an antibody Fab structure. There are, however, some important differences, particularly in the $\mathrm{C} \alpha$ domain, which are consistent with its relatively lower sequence homology to Ig $\mathrm{C}$ domains. An Ig $\mathrm{C}$ domain can be described as having two $\beta$-sheets, a 'top' sheet composed of three strands $(\mathrm{c}, \mathrm{f}, \mathrm{g})$, and a 'bottom' sheet composed of four $(a, b, e, d)$. In TCR C $\alpha$, the top strands are too far apart to form a $\beta$-sheet, in part exposing the bottom sheet to solvent. There are additional departures from Ig structure in the interdomain pairing between $\mathrm{C} \alpha$ and $\mathrm{C} \beta$.

The complex, at $3.4 \AA$, with class I MHC and bound peptide antigen (Fig. 2) shows the orientation of TCR within the complex ${ }^{3}$. The antigen-binding cleft of MHC consists of an eight-stranded $\beta$-sheet 'platform' topped by two parallel $\alpha$ helices. The relatively flat TCR combining site interacts with the exposed residues of the antigen and also the two parallel $\alpha$-helices of MHC; the long axes of the binding surfaces are not quite parallel. The CDR3s of $V \alpha$ and $\beta$ form a deep cleft, and are principally involved in contacting the peptide antigen, while CDRs 1 and 2 also appear to interact largely with the MHC parallel $\alpha$-helices. It will be well worth solving several different TCR-MHC-antigen complexes, varying MHC class and allele, antigen (and specific TCR), to understand the general principles of recognition.

Our new reference to the TCR structure demarcates a line conceptually dividing the T-cell activation pathway. On one side is the basis for the recognition of MHC presenting antigen, now well illuminated by Garcia et al. ${ }^{3}$ On the other side is a structural understanding of how that recognition is transduced into a response by the T-cell. The TCR does not act alone, on either side of this line. While the TCR is responsible for the specificity of antigen-plus-MHC recognition, another cell surface molecule, either CD4 or CD8 is responsible for distinguishing between $\mathrm{MHC}$ classes. Furthermore, TCR on its own cannot transduce T-cell activation-the cytoplasmic domain of TCR seems to be too short. Instead, associated membrane proteins of the TCR complex are required: three transmembrane chains of CD3, and the $\zeta$ chains (sometimes considered as part of $\mathrm{CD} 3$ ). Binding of antigen-MHC to TCR results in phosphorylation of tyrosine resides in the cytoplasmic domains of these other proteins, which in turn feeds into a phospholipase $\mathrm{C}$ signal transduction pathway. CD3 and TCR potentially could interact through their membrane-spanning domains, but there is also indirect evidence for an interaction of an extracellular region of the TCR C $\alpha$ chain with the $\zeta$ chain. Garcia et al. note that this region of the $\mathrm{C} \alpha$ chain, corresponding to the top, atypical $c$, $\mathrm{f}$ and $\mathrm{g}$ strands, may be flexible, which they propose to be important for interaction with the $\zeta$ chain. The association with the CD3 and $\zeta$ chains necessary for assembly of the complex and signal transduction is a particularly interesting focus for further structural work, even though the possibility that some aspects of this interaction take place within the membrane makes the prospects daunting.

The recognition of $\mathrm{MHC}$-antigen represents more than just a useful way to divide T-cell activation into discrete parts. Recognition can be viewed as the step after which signal transduction amplifies and reinforces itself in a maze of interactions within the cell. Moreover, recognition occurs on the outside of the cell, and may thus be amenable to modification. Since recognition of an infected cell, transplant rejection, and misrecognition of a normal cell in autoimmune disease, among others, are processes 'funnelled' through the interaction of TCR with MHC-antigen, designed drugs that either boost or inhibit TCR recognition could potentially treat a very wide range of diseases.

1. Zinkernagel, R.M. \& Doherty, P.C. Nature 251, 547-548 (1974),
2. Stem, L.J \& Wiley, D.C. Structure 2, 245-251 (1994).

3. Garcia, K.C. et al. Science 274, 209-219 (1996). 